\title{
Genome-Wide Association Study of Sleep Disturbances in Depressive Disorders
}

\author{
Lindsay M. Melhuish Beaupre ${ }^{a, d}$ Vanessa F. Gonçalves ${ }^{b, d} \quad$ Clement C. Zai $^{a-d}$ \\ Arun K. Tiwari $^{b}$, d Ricardo S. Harripaul ${ }^{a, e}$ Deanna Herbert ${ }^{d}$ Natalie Freeman ${ }^{d}$ \\ Daniel J. Müller ${ }^{a, b, f}$ James L. Kennedy $a, b, d$ \\ ${ }^{\mathrm{a}}$ Institute of Medical Sciences, University of Toronto, Toronto, ON, Canada; ${ }^{\mathrm{b}}$ Department of Psychiatry, University \\ of Toronto, Toronto, ON, Canada; ' Laboratory Medicine and Pathobiology, University of Toronto, Toronto, ON, \\ Canada; ${ }^{\mathrm{d}}$ Molecular Brain Science Research Department, Campbell Family Mental Health Research Institute, \\ Centre for Addiction and Mental Health, Toronto, ON, Canada; ${ }^{e}$ Molecular Neuropsychiatry and Development Lab, \\ Campbell Family Mental Health Research Institute, Centre for Addiction and Mental Health, Toronto, ON, Canada; \\ fPharmacogenetics Research Clinic, Campbell Family Mental Health Research Institute, Centre for Addiction and \\ Mental Health, Toronto, ON, Canada
}

\section{Keywords}

Genome-wide association study · Sleep disturbances ·

Depression · Genetics

\begin{abstract}
Sleep disturbance affects about $75 \%$ of depressed individuals and is associated with poorer patient outcomes. The genetics in this field is an emerging area of research. Thus far, only core circadian genes have been examined in this context. We expanded on this by performing a genome-wide association study (GWAS) followed by a preplanned hypothesis-driven analysis with 27 genes associated with the biology of sleep. All participants were diagnosed by their referring physician, completed the Beck Depression Inventory (BDI), and the Udvalg for Kliniske Undersogelser Side Effect Rating Scale at baseline. Our phenotype consisted of replies to 3 questions from these questionnaires. From standard GWAS chip data, imputations were performed. Baseline total BDI scores $(n=364)$ differed significantly between those
\end{abstract}

(c) 2020 S. Karger AG, Basel

karger@karger.com

www.karger.com/mnp with and those without sleep problems. We were unable to find any significant GWAS hits although our top hit was for changes in sleep and an intergenic marker near SNX18 ( $p=$ $\left.1.06 \times 10^{-6}\right)$. None of the markers in our hypothesis-driven analysis remained significant after applying Bonferroni corrections. Our top finding among these genes was for rs 13019460 of Neuronal PAS Domain Protein 2 with changes in sleep $(p=0.0009)$. Overall, both analyses were unable to detect any significant associations in our modest sample though we did find some interesting preliminary associations worth further exploration.

c) 2020 S. Karger AG, Basel

Depression is a debilitating mood disorder that affects $11-16 \%$ of the population throughout their lifetime [13]. Most individuals with depression experience substantial impairment, making it the second leading cause of years lived with a disability $[2,4]$. One of the core symptoms of depression, as stated by the Diagnostic and Statistical Manual of Mental Disorders (5th ed), is hyper-

Dr. James L. Kennedy

Molecular Brain Science Research Department Centre for Addiction and Mental Health 250 College Street, Toronto, ON, M5T 1R8 (Canada) jim.kennedy@camh.ca 
somnia and/or insomnia [5]. In fact, about $75 \%$ of these individuals experience sleep disturbances [6]. These disturbances are known to have marked effects on the course of the disorder and treatment. For example, irregular sleep physiology is associated with a greater risk of relapse, a greater rate of requiring pharmacological therapy, not responding to psychotherapy and a higher chance of not experiencing remission [7-11]. They have also been found to be a prodromal symptom of depressive disorders [12]. For example, The Munich Vulnerability study found that about a quarter of the high-risk individuals studied (those that have a first degree relative with an affective disorder) experienced changes in their sleep physiology for 10 years leading up to the onset of an affective disorder [13].

Genetics of sleep in depression is an emerging area of research. Thus far, there have been only a limited number of studies done investigating the genetics of sleep disturbances in depression. Of these few studies, all have focused on circadian genes because of their involvement in the regulation of sleep-wake cycles [14]. The only positive findings thus far have been for variants of TIMELESS and CLOCK [15-17]. For TIMELESS, the minor "A" allele of rs7486220 and the "AA" haplotype of rs2291738rs7486220 have been linked to daytime fatigue in females, whereas the " $T$ " allele of rs1082214 was linked to earlier morning awakenings in males. The findings for CLOCK are mixed as 1 study has found an association with rs 1801260 and initial insomnia while another was unable to replicate these findings $[15,17]$.

Genetic studies in MDD research have mainly focused on disease risk. A recent mega-analysis performed by the Major Depressive Disorder Working Group of the Psychiatric Genomics Consortium, combined 9 samples for a total of 18,759 unrelated subjects of European ancestry $(9,240$ cases and 9,519 controls), but was unable to detect any genome-wide significant associations. A follow-up study done on a large replication sample ( $n=9,238 \mathrm{MDD}$ subjects and $n=8,039$ controls) also failed to identify any significant loci [18]. More recently, Hyde et al. [19] identified 15 genetic loci associated with a risk of self-reported major depression in $n=307,354$ individuals of European ancestry ( $n=65,607$ cases and $n=231,747$ controls from 23 and $\mathrm{Me}$ ). The most significant finding was for rs2806933 of OLFM4, a gene encoding for olfactomedin-4, that has been previously associated with childhood obesity. The marker rs768705, which is located at a locus containing myocyte enhancer factor $2 \mathrm{C}$ and TMEM161B (transmembrane protein 161B) was also significant [19, 20]. A second marker at myocyte enhancer factor $2 \mathrm{C}$ -
Table 1. Genes included in the hypothesis-driven analysis

\begin{tabular}{lll}
\hline CLOCK & TIMELESS & PER1 \\
PER2 & PER3 & CRY1 \\
CRY2 & BMAL1 & ARNTL2 \\
NPAS2 & RORA & RORB \\
RARA & HCRT & HCRTR1 \\
HCRTR2 & MCHR1 & MCHR2 \\
SIRT1 & MTNR1A & MTNR1B \\
KCNH4 & AANAT & USP2 \\
OPN4 & ADORA2A & ADA \\
\hline
\end{tabular}

CLOCK, Clock Circadian Regulator; TIMELESS, Timeless Circadian Regulator; PER1, Period Circadian Regulator 1; PER2, Period Circadian Regulator 2; PER3, Period Circadian Regulator 3; CRY1, Cryptochrome Circadian Regulator 1; CRY2, Cryptochrome Circadian Regulator 2; BMAL1, Aryl Hydrocarbon Receptor Nuclear Translocator Like; ARNTL2, Aryl Hydrocarbon Receptor Nuclear Translocator Like 2; NPAS2, Neuronal PAS Domain Protein 2; RORA, RAR Related Orphan Receptor A; RORB, RAR Related Orphan Receptor B; RARA, Retinoic Acid Receptor Alpha; HCRT, Hypocretin Neuropeptide Precursor; HCRTR1, Hypocretin Receptor 1; HCRTR2, Hypocretin Receptor 2; MCHR1, Melanin Concentrating Hormone Receptor 1; MCHR2, Melanin Concentrating Hormone Receptor 2; SIRT1, Sirtuin 1; MTNR1A, Melatonin Receptor 1A; MTNR1B, Melatonin Receptor 1B; KCNH4, Potassium Voltage-Gated Channel Subfamily H Member 4; AANAT, Aralkylamine N-Acetyltransferase; USP2, Ubiquitin Specific Peptidase 2; OPN4, Opsin 4; ADORA2A, Adenosine A2a Receptor; ADA, Adenosine Deaminase.

TMEM161B, rs10514299, showed a promising association with risk to MDD in a replication sample [19]. None of these 3 genes has been reported to be associated with sleep measures in depressive disorders.

To our knowledge, this current study is the first to perform a genome-wide association study (GWAS) investigating the genetics of sleep disturbances in depressive disorders. Our hypothesis-free approach investigated the genetic basis of each of 3 items from our questionnaires, namely, changes in sleep, increased sleep, and decreased sleep, in depressed individuals. As a following step, we apply a hypothesis-driven approach to test the role of selected markers in key genes (Table 1) involved in the biology of sleep using the same sample and measures of sleep disturbances from step 1. In this approach, we are able to examine more closely the key markers in the biology of sleep, including the TIMELESS gene.

\section{Methods}

Subjects

The sample will be a subset of the IMPACT study $(n>11,000)$ [21]. This study was originally designed to examine the association of genetic variants with treatment response and side effects of antidepressants and antipsychotics. Participants were asked to pro- 
vide a saliva sample for genetic analyses and fill out a series of questionnaires depending on their diagnoses. All individuals filled out the Beck Depression Inventory (BDI) [22] and the Udvalg for Kliniske Undersogelser (UKU) Side Effect Rating Scale [23]. These individuals were followed prospectively for $\sim 8$ weeks and asked to fill out the same questionnaires at a 1-month follow-up and a 2-month follow-up. The diagnoses were made by the referring physician and included: major depressive disorder, depression, depressive episode NOS, depression with psychosis, unipolar and dysthymic disorder. The analyses in this report focused on individuals that had a BDI score $>19$ at baseline to represent moderate to severe depression [24], and were restricted to European ancestry to reduce population stratification and to allow for the greatest sample size. The ratio of males to females was 110:256. This study was approved by the Centre for Addiction and Mental Health $(\mathrm{CAMH})$ Research Ethics Board. All individuals provided informed consent.

\section{Genotyping}

Genomic DNA was extracted at CAMH from saliva collected in Oragene saliva kits using the magnetic bead DNA extraction protocol on the chemagic Magnetic Separation Module I (Perkin Elmer, Waltham, MA, USA) automated DNA extractor as per the manufacturer's standard protocol. A subset $(n=2,116)$ of individuals was genotyped using the Infinium Omni 2.5-8 chip at The Centre for Applied Genomics at The Hospital for Sick Children, Toronto. This chip contains $\sim 2.5$ million markers well-suited for genotyping individuals of European ancestry [25].

\section{Quality Control}

Genetic quality control (QC) was done on 2,116 individuals who were examined with the Illumina Infinium Omni 2.5-8 chip array using PLINK (version 1.9) [26] and standard protocols described in other GWAS studies [27-29]. The participants in our sample were checked for sex discordance, individual genotype missingness (which was restricted to $2 \%$ ), heterozygosity rate, relatedness (identity-by-descent), and genetic ancestry to compare with self-reported ancestry. For heterozygosity rate, individuals were removed if they were \pm 3 SDs away from the mean to avoid potential confounding of samples or inbreeding. A principal component analysis (PCA) was done to measure self-reported ancestry. To do this, we plotted our sample versus the 1,000 Genomes Phase 3 reference population [30] using R [31]. After individual QC and selecting for European ancestry by the initial PCA analysis, we were left with $n=1,733$ before the final PCA analysis. Individuals considered to be ancestral outliers were removed from the study if they were \pm 6 SDs from the mean [32]. After this final PCA step, we were left with $n=1,098$ individuals and next we selected those with a clinical depressive disorder leaving 623 people. Finally, we excluded individuals with a BDI score $<20$, and we were left with 366 individuals.

Genome-wide marker QC was also done to check for significant deviation from Hardy-Weinberg equilibrium $\left(p<5 \times 10^{-8}\right)$, minor allele frequency $(<1 \%)$, and genotype call rates $>98 \%$ for both markers and individuals. After conducting these QC procedures, we were left with 1,295,909 markers.

\section{Imputations}

Whole-genome imputations were conducted using IMPUTE version 2.2 in 5,000,000 Mb segments per chromosome after pre- phasing was performed with SHAPEIT2 and the 1,000 Genomes reference panel (Phase 3, NCBI build 37) [30, 33, 34]. After performing the imputations, we conducted the same marker QC procedures as described above [27-29]. This time, we excluded markers with a minor allele frequency $<0.05$ in the European population, $<95 \%$ genotyping rates, and variants that had an info score threshold of $\geq 0.8$ [35]. After applying all QC procedures we were left with 5,932,083 markers.

\section{Sleep Measures}

The sleep measures of interest were questions 16 in the BDI (changes in sleep) and in the UKU 1.7 (increased sleep) and 1.8 (decreased sleep). Both the BDI and the UKU were given to participants at baseline, at the 1-month follow-up and 2-month follow-up. Question 16 from the BDI asks about "Changes in Sleeping Pattern" over the course of 2 weeks with $0=$ "I have not experienced any change in my sleeping pattern," $1 \mathrm{a}=$ "I sleep somewhat more than usual," $1 \mathrm{~b}=$ "I sleep somewhat less than usual," $2 \mathrm{a}=$ "I sleep a lot more than usual," $2 \mathrm{~b}=$ "I sleep a lot less than usual," $3 \mathrm{a}=$ "I sleep most of the day," $3 \mathrm{~b}=$ "I wake up 1-2 h early and can't get back to sleep." The question is coded such that individuals that choose $1 \mathrm{a}$ or $\mathrm{b}$ get a score of 1 , those who choose $2 \mathrm{a}$ or $\mathrm{b}$ receive a score of 2 and those who choose $3 \mathrm{a}$ or $\mathrm{b}$ get a score of 3. Question 1.7 of the UKU asks regarding an "Increased Duration of Sleep" and 1.8 asks about a "Reduced Duration of Sleep" over the last 3 days. Both questions are provided with a Likert scale from 0 to 3 with $0=$ "No side effects," $1=$ "Mild side effects that do not interfere with the patient's performance," 2 = "Side effects that interfere moderately with the patient's performance" and 3 = "Side effects that interfere markedly with the patient's performance." All 3 questions were recoded into binary data with 0 representing those who scored a 0 on the question of interest and 1 representing those who scored a 1,2 , or 3 on the question of interest.

\section{Statistics}

A binary logistic regression was performed using PLINK (version 1.9) [26] independently for each of the 3 questions of interest for both the GWAS and hypothesis-driven data, using an additive model of inheritance for all single nucleotide polymorphism (SNP) data. Results were considered significant in the GWAS data if they had a $p$ value $<5.0 \times 10^{-8}$. Nyholt test corrections for linkage disequilibrium (LD) were applied to the hypothesis-driven data [36]. After correcting for 1953 ("sleep") SNPs in the hypothesis-driven data, a $p$ value of 0.000025 was considered significant. Chi-square analysis was performed to determine whether sex should be analyzed as a covariate. An independent sample $t$ test was conducted to determine if age and baseline BDI score should be analyzed as covariates.

\section{Results}

After removing all individuals that did not have a depressive disorder and did not complete the BDI after performing imputations and QC procedures, we were left with $n=364$ participants. Table 2 for clinical demographics. Note that analyses were limited to those with Euro-
Melhuish Beaupre et al. 


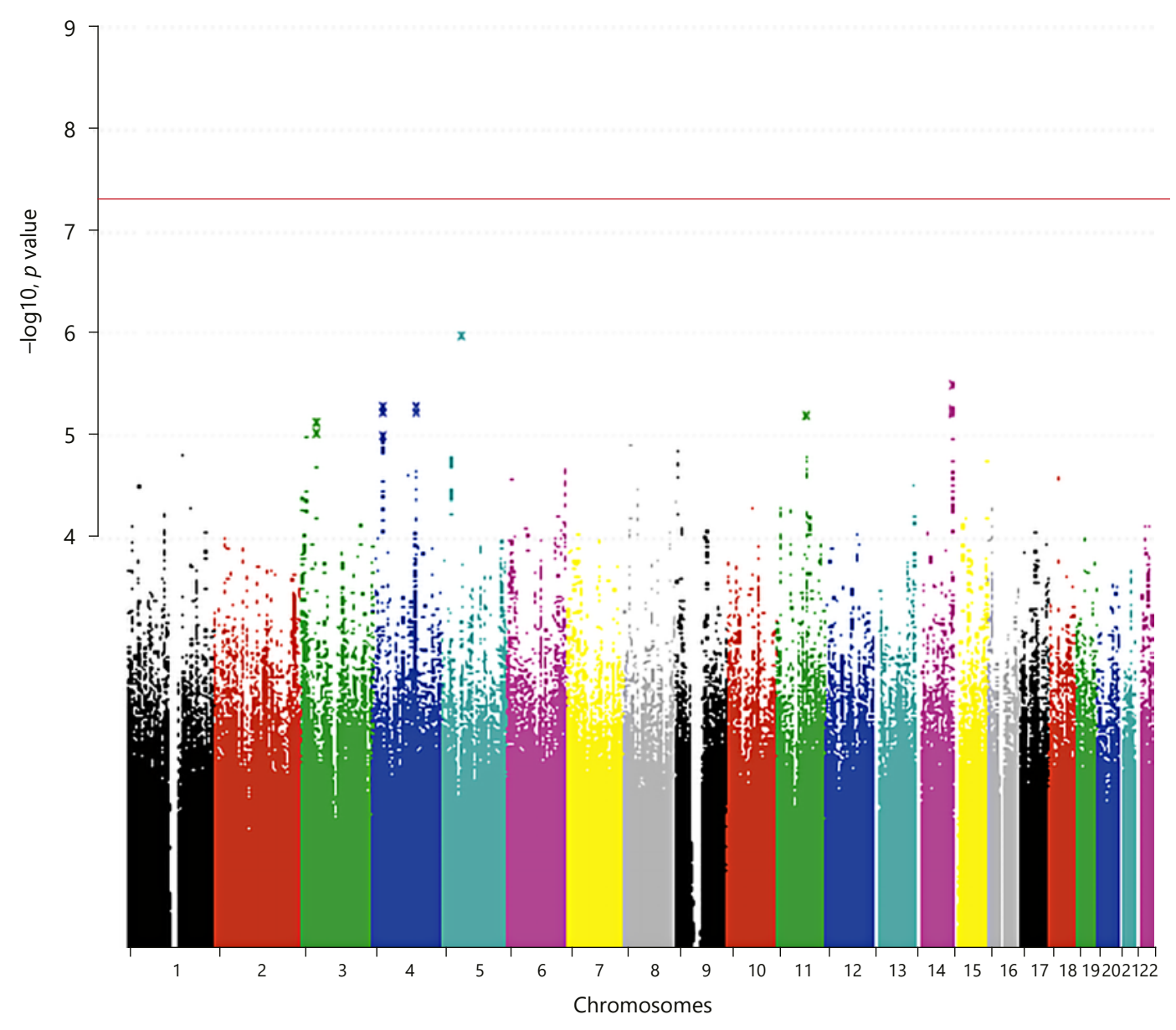

Fig. 1. Manhattan plot for the BDI 16.

Table 2. Clinical demographics

\begin{tabular}{|c|c|c|c|c|c|c|c|}
\hline & \multicolumn{2}{|l|}{ BDI 16} & \multicolumn{2}{|l|}{ UKU 1.7} & \multicolumn{2}{|l|}{ UKU 1.8} & \multirow[t]{3}{*}{ Total } \\
\hline & \multicolumn{2}{|c|}{ sleep problems } & \multicolumn{2}{|c|}{ sleep problems } & \multicolumn{2}{|c|}{ sleep problems } & \\
\hline & absent & present & absent & present & absent & present & \\
\hline Sample size & 36 & 329 & 128 & 223 & 144 & 219 & 366 \\
\hline Gender, male:female & $10: 26$ & $100: 229$ & 43:85 & $62: 161$ & $37: 107$ & $71: 148$ & $110: 256$ \\
\hline Current age, mean (SD) & $44.67(15.23)$ & $47.34(15.49)$ & $47.4(15.57)$ & $47.23(15.34)$ & $46.5(15.21)$ & $47.74(15.45)$ & $47.01(15.5)$ \\
\hline BDI score, mean (SD) & $30.11(7.5)$ & $33.7(8.77)$ & $31.78(8.22)$ & $34.42(8.86)$ & $31.85(8.42)$ & $34.42(8.73)$ & $33.33(8.71)$ \\
\hline Concurrent psychiatric diagnoses, $n(\%)$ & $24(66.7)$ & $193(58.7)$ & $81(63.3)$ & $136(61)$ & $87(60.4)$ & $141(64.4)$ & $231(63.1)$ \\
\hline Concurrent anxiety diagnoses, $n(\%)$ & $21(58)$ & $173(52.6)$ & $73(57)$ & $116(52)$ & $72(50)$ & $121(55.2)$ & $195(53.3)$ \\
\hline
\end{tabular}

Some people did not fill out all of the questions, hence why the sample size varies.

BDI, Beck Depression Inventory; UKU, Udvalg for Kliniske Undersogelser Side Effect Rating Scale. 


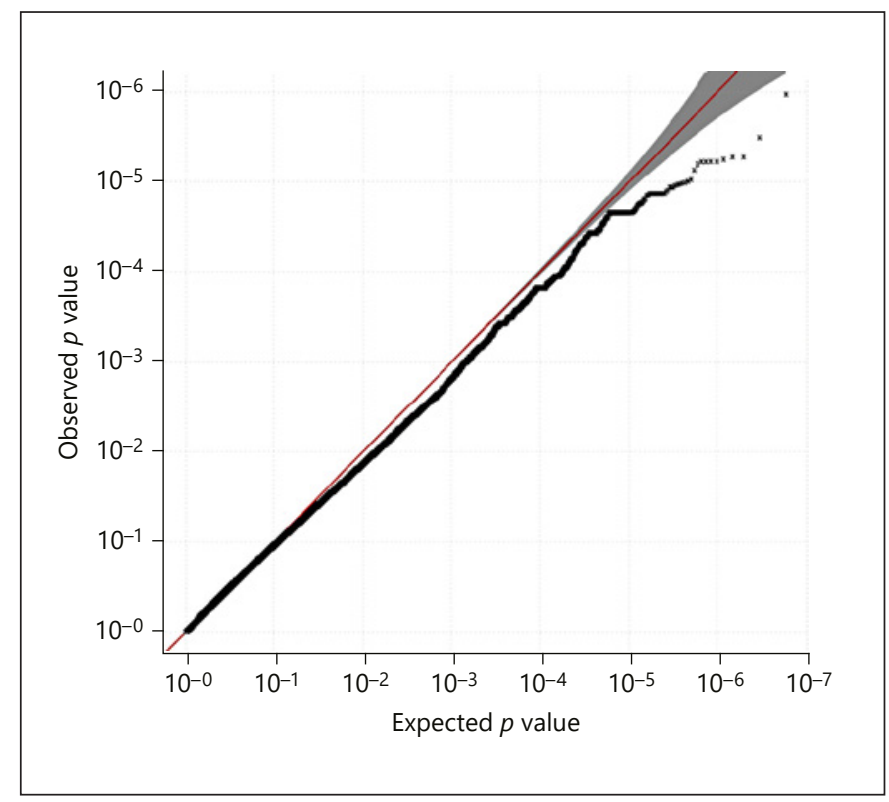

Fig. 2. QQ plot for BDI 16.

pean genetic ancestry. The baseline total BDI score was significantly different between individuals with or without sleep problems for the BDI 16 (changes in sleep; $t=$ $-2.68, \mathrm{df}=46.15, p=0.01$ ), UKU 1.7 (increased sleep; $t=$ $-2.81, \mathrm{df}=281.31, p=0.005)$, and UKU 1.8 (decreased sleep; $t=-28, \mathrm{df}=313.81, p=0.005)$. There were no significant correlations between age for either the BDI 16 $(t=-1, \mathrm{df}=43.31, p=0.32), \mathrm{UKU} 1.7(t=0.96, \mathrm{df}=261.53$, $p=0.92)$, or UKU $1.8(t=-0.76, \mathrm{df}=309.47, p=0.45)$. There was also no significant sex effect found for the BDI $16\left(\chi^{2}=0.106, \mathrm{df}=1, p=0.75\right)$, UKU $1.7\left(\chi^{2}=1.3, \mathrm{df}=1\right.$, $p=0.25)$, or UKU $1.8\left(\chi^{2}=1.88, \mathrm{df}=1, p=0.17\right)$. Thus, we only included baseline BDI score as a covariate in our analyses, although this did not influence the results.

\section{Hypothesis-Free GWAS}

From the hypothesis-free approach, none of the SNPs was significantly associated with sleep disturbances with any of the questions of interest (Fig. 1-6). There were some nominally significant associations, for example, the top finding from our analyses for changes in sleep (BDI 16) was for rs 2565010 , located $44 \mathrm{~kb} 3^{\prime}$ of $S N X 18$ (OR 0.22, $p=1.06$ $\left.\times 10^{-6}\right)$ and 2 SNPs in the N-deacetylase and N-sulfotransferase 3 (NDST3) coding region, rs7436139 and rs6835510 $\left(\mathrm{OR} 0.26, p=5.21 \times 10^{-6}\right.$ and OR $0.19, p=$ $5.9 \times 10^{-6}$, respectively). Other top hits were intergenic regions (online suppl. Table 1; for all online suppl. material, see www.karger.com/doi/10.1159/000505804). For the in- creased sleep (UKU 1.7), our top findings were for 19 intergenic SNPs (online suppl. Table 2), as well as rs57288534 of RASGEF1C (OR 3.31, $\left.p=8.82 \times 10^{-6}\right)$ and 2 SNPs in ryanodine receptor 2 (RYR2), rs 10802610 and $\mathrm{rs} 12041884$ (OR $0.4, p=8.9 \times 10^{-6}$ and OR 0.4, $p=9.45 \times 10^{-6}$, respectively). Lastly, our top findings for decreased sleep (UKU 1.8) were for rs4076190 of ANTXR1 (OR 0.46, $p=2.77 \times 10^{-6}$ ) and several other intergenic SNPs (online suppl. Table 3).

\section{Hypothesis Driven}

None of our SNPs was significantly associated with sleep disturbances on any of the 3 questions of interest after applying Nyholt corrections for multiple testing. Our top findings for changes in sleep were 17 variants in Neuronal PAS Domain Protein 2 (NPAS2), with the top hit being rs13019460 (OR 2, $p=0.0009$ ). Other nominal findings were 6 SNPs in Period Circadian Regulator 2 (PER2; top hit: rs78639475, OR 2, $p=0.0067$ ) and rs2234975 of Sirtuin 1 (SIRT1; OR 2.4, $p=0.0068$ ) with increased sleep and 14 SNPs in hypocretin/orexin receptor 2 (HCRTR2) and decreased sleep, with the top hit being rs3134696 (OR 1.7, $p=0.0062$ ).

\section{Discussion}

First, we performed a GWAS investigating the genetics of sleep disturbances in depressive disorders in the CAMH IMPACT sample. Following this, we tested the association of 5,895 SNPs related to the biology of sleep with 3 measures of sleep disturbances in the same depression sample. We were unable to detect any genome-wide associations in our hypothesis-free analyses, though we did find some nominally significant $\left(p\right.$ value $<9.99 \times 10^{-6}$ ) associations that warrant further investigation. The coding region of the NDST3, which is involved in heparin biosynthesis and heparan sulfate metabolism [37, 38], was the top hit in terms of known coding regions. It is expressed both in the fetal and adult brains [39]. Both heparin and haparan sulfate play an important role in many vital cellular processes including cell growth and signaling [40]. Lencz et al. [41] found that rs11098403 of NDST3 was previously associated with schizophrenia and bipolar disorder in an Ashkenazi Jewish sample. Although neither of the SNPs identified in our study is in LD with the SNP found in Lencz et al. [41], it does provide some support that the gene itself might be implicated in sleep disturbances in depressive disorders. Another promising hit was for 2 variants in the RYR2. Ryanodine receptors play a role in the release of calcium from calci-
Melhuish Beaupre et al. 


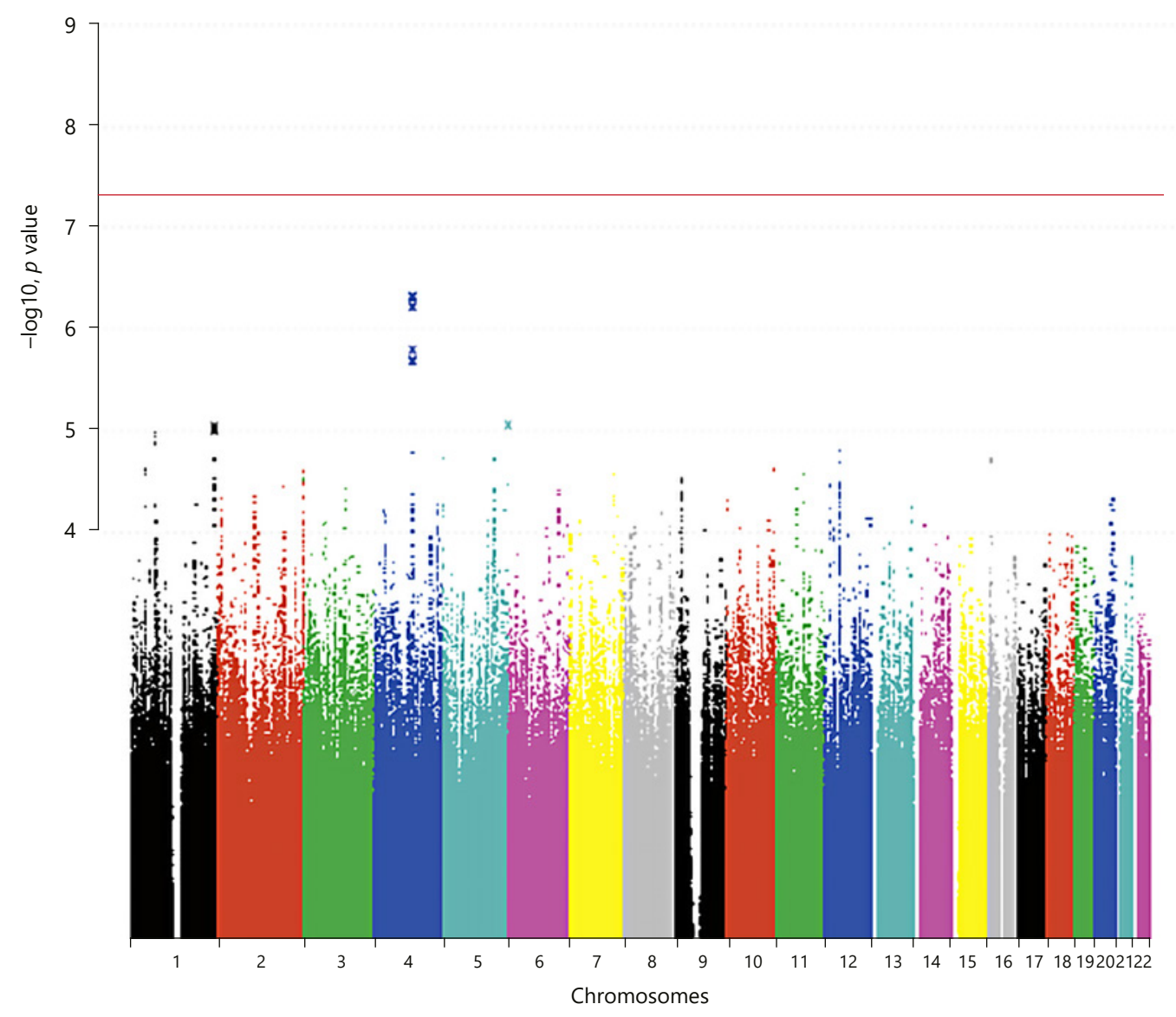

Fig. 3. Manhattan plot of the UKU 1.7.

um channels in mammalian skeletal and cardiac muscle [42]. Numerous mutations in this gene have been associated with catecholaminergic polymorphic ventricular tachycardias, which may result in sudden cardiac death $[43,44]$. More recent research has suggested that ryanodine receptors are regulated by the CLOCK/BMAL1 complex [45]. CLOCK and BMAL1 proteins are found in the suprachiasmatic nucleus (SCN), the master clock of the body. The SCN contains a set of genes responsible for regulating the body's circadian rhythms and sleep-wake cycle [46]. As such, it is plausible that RYR2 plays a role in the SCN and regulating sleep-wake cycles, although extant research is limited and further work is warranted.

In our secondary, hypothesis-driven analyses, we were unable to detect any significant associations. Our top hit was for a variant (rs13019460) in NPAS2, a core circadian gene found in the SCN. NPAS2 is considered homologous to the CLOCK gene. It has been found that in CLOCKdeficient mice, NPAS2 will form a heterodimer with

GWAS of Sleep Disturbances in Depression

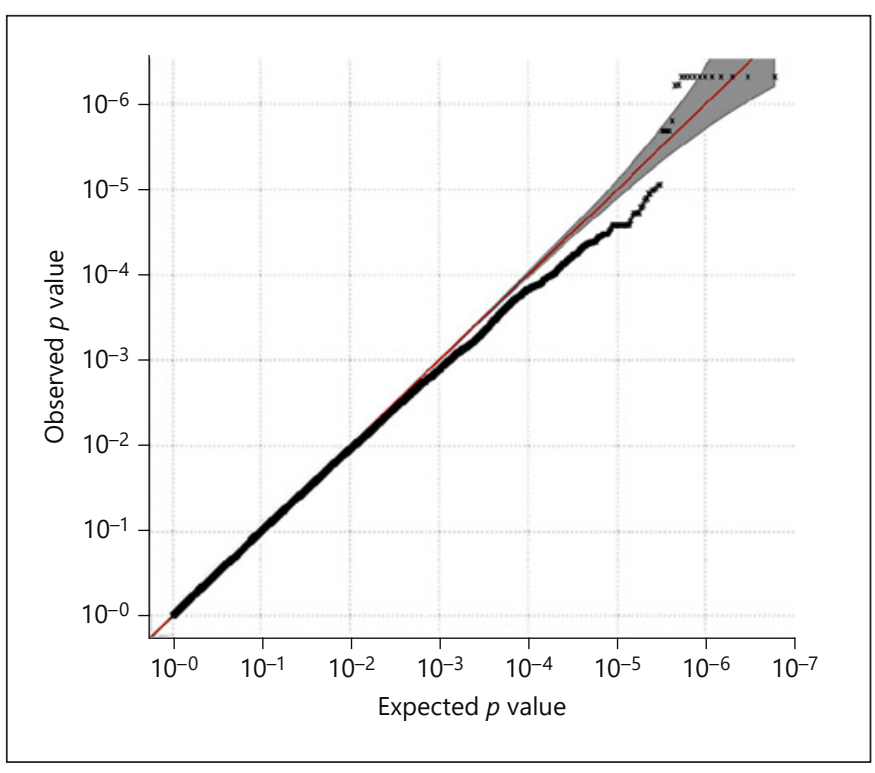

Fig. 4. QQ plot of the UKU 1.7.

Mol Neuropsychiatry 2019;5(suppl 1):34-43 DOI: $10.1159 / 000505804$ 


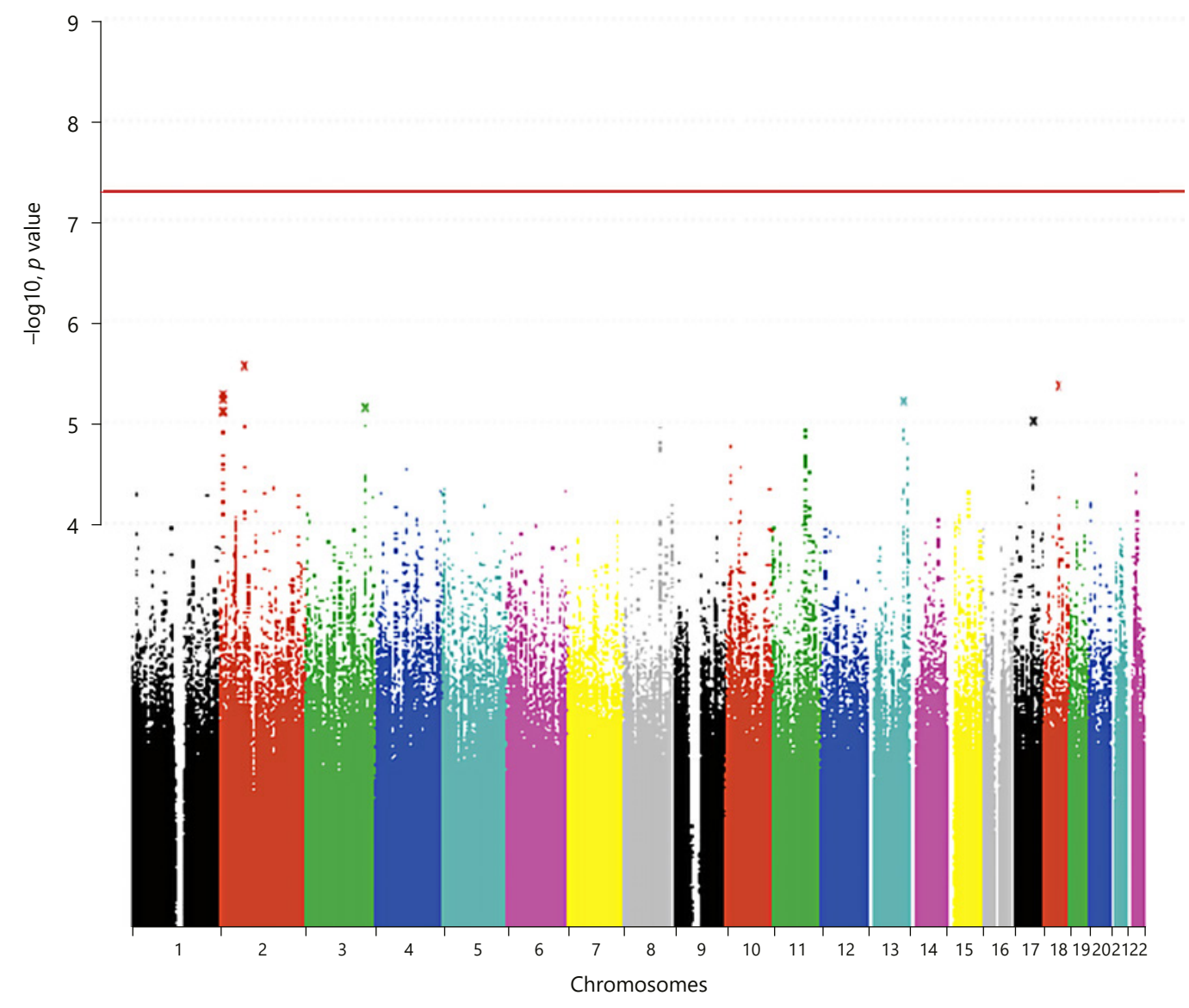

Fig. 5. Manhattan plot for the UKU 1.8 .

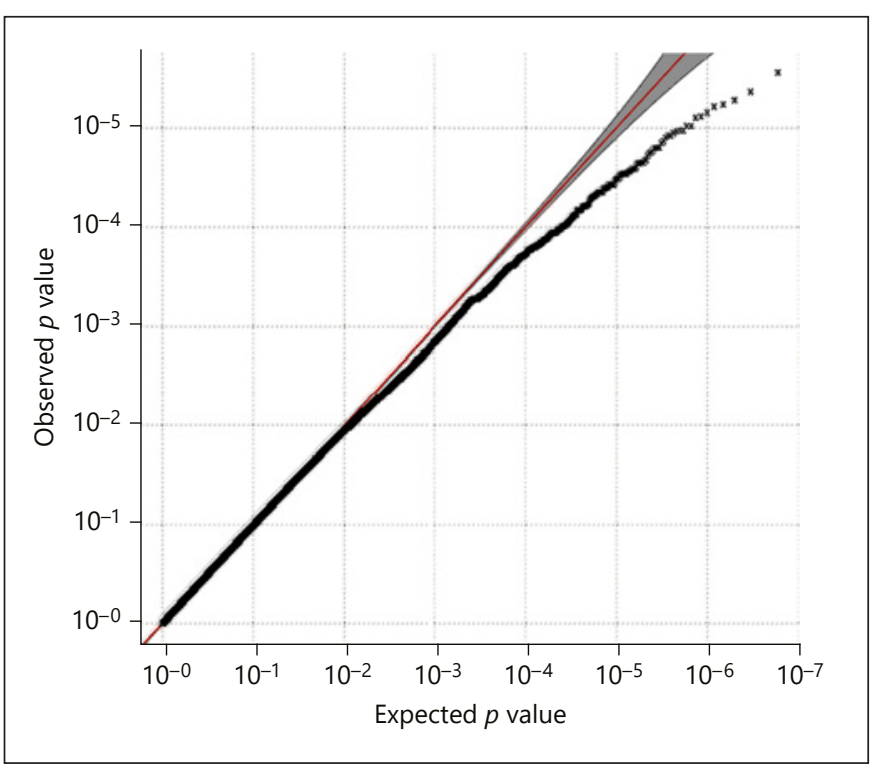

Fig. 6. QQ plot for the UKU 1.8.
BMAL1, which allows the SCN to maintain circadian rhythmicity [47]. Furthermore, Evans et al. [48] found an association between rs3768984 of NPAS2 and objectively measured sleep parameters using actigraphy. Soria et al. [49] also implicated another variant of the gene, rs11123857 in depression. Although neither of these SNPs is in LD with our SNP of interest, taken together, the evidence suggests that NPAS2 could play a role in sleep disturbances among depressed individuals. PER2, another core circadian gene has had several variants associated with depression susceptibility versus mental resilience in a sample of $n \sim 430$ individuals, although no variant in the gene was associated with depression in case-control analyses [50,51]. PER2, like NPAS2 is part of the core clock machinery, as it is part of the positive and negative transcriptional feedback loops in mammals that regulates the 24-h (circadian) cycle in the SCN [52, 53]. As such, it seems plausible that PER2 plays a role in sleep disturbances in depressed individuals. However, 
Utge et al. [16] were unable to find any association with variants in PER2 and sleep disturbances in depression in either males or females. We also observed nominal promising results for rs 2234975 , located in the untranslated region of SIRT1. Although SIRT1 is not considered a core circadian gene, it has been shown to bind to the CLOCKBMAL1 complex and directly regulate PER2, suggesting it plays a role in the regulation of circadian rhythms [54]. Therefore, it seems like a promising gene to study in this context. Lastly, we also found nominal associations for several variants in the HCRTR2. This gene has several lines of evidence supporting its role in the sleep-wake cycle. Lin et al. [55] were the first to discover that disruption of HCRTR2 led to narcolepsy in canines. There is also evidence supporting the involvement of hypocretin/orexin in promoting wakefulness [56]. More recently, a GWAS study investigating sleep traits identified rs3122163, a variant near HCRTR2 to be linked to shorter sleep duration [57]. Although this SNP is not in LD with any of the SNPs found in our study, all of the evidence together suggests that HCRTR2 may play a role in sleep disturbances.

A limitation of this study is that we have a modest sample size, which means that our results could be false-positive results. Second, our study is based on subjective measures, which can be distorted by recall bias or other biases in the participants. Lastly, a confound may lie in the fact that most participants had comorbid psychiatric diagnoses, as in the case in most samples of psychiatric disorders.

Overall, the research into the genetics of sleep disturbances is limited. As such, we sought out to perform a hypothesis-free GWAS study, followed by a secondary hypothesis-driven analysis to explore biologically informed genetic marker associations with sleep disturbances in a subset of the CAMH IMPACT study. We were unable to detect any significant associations in any of our analyses. We provide potentially useful preliminary data and methodological approaches to inform future investigations of this important area.

\section{Acknowledgments}

We would like to thank the CAMH IMPACT clinical team, including Nicole Braganza for collecting the data on the participants used in this study.

\section{Statement of Ethics}

The research was conducted ethically in accordance with the CAMH Ethics Board.

\section{Disclosure Statement}

J.L.K. is a member of the Scientific Advisory Board of Myriad Genetics (unpaid) and has several patents pertaining to pharmacogenetic testing for psychiatric illnesses. All other authors have no conflicts of interest to declare.

\section{Funding Source}

This study was funded by an Anonymous donor and the Larry and Judy Tanenbaum Family Foundation.

\section{Author Contributions}

L.M.M.B.: performed the imputations, data analysis, interpretation of results, and the write-up of the manuscript. V.F.G.: provided support and guidance on performing the imputations, data analysis, and interpretations of the results. She also helped draft the manuscript. C.C.Z. and A.K.T.: provided guidance on the data analysis, interpretation of results, and the write-up of the manuscript. D.H. was involved in the clinical data collection, analysis of the data, and editing of the manuscript. N.F. was involved in the generation of data from the lab, methods design, and the drafting and editing of the manuscript. D.J.M. helped with the data analysis, interpretation of results, and editing of the manuscript. J.L.K. oversaw and assisted with the data analysis, interpretation of the results, and the drafting of the manuscript.

\section{References}

1 Bromet E, Andrade LH, Hwang I, Sampson NA, Alonso J, de Girolamo G, et al. Crossnational epidemiology of DSM-IV major depressive episode. BMC Med. 2011 Jul;9: 90.

2 Kessler RC, Berglund P, Demler O, Jin R, Koretz D, Merikangas KR, et al. The Epidemiology of Major depressive disorder: results from the National Comorbidity Survey Replication (NCS-R). JAMA. 2003 Jun;289(23):3095105.
3 Smetanin P, Stiff D, Briante C, Ahmad S, Khan M. The Life and Economic Impact of Major Mental Illnesses in Canada: 2011 to 2041. RiskAnalytica, on behalf of the Menal Health Commission of Canada 2011. 2011.

4 Vos T, Barber RM, Bell B, Bertozzi-Villa A, Biryukov S, Bolliger I, et al.; Global Burden of Disease Study 2013 Collaborators. Global, regional, and national incidence, prevalence, and years lived with disability for 301 acute and chronic diseases and injuries in
188 countries, 1990-2013: a systematic analysis for the Global Burden of Disease Study 2013. Lancet. 2015 Aug;386(9995): 743-800.

5 American Psychiatric Association. Diagnostic and statistical manual of mental disorders. 5th Editio. Washington (DC): American Psychiatric Association; 2013.

6 Nutt D, Wilson S, Paterson L. Sleep disorders as core symptoms of depression. Dialogues Clin Neurosci. 2008;10(3):329-36. 
7 Pigeon WR, Hegel M, Unützer J, Fan MY, Sateia MJ, Lyness JM, et al. Is insomnia a perpetuating factor for late-life depression in the IMPACT cohort? Sleep. 2008 Apr;31(4):481-8.

8 Van Londen L, Molenaar RP, Goekoop JG, Zwinderman AH, Rooijmans HG. Three- to 5 -year prospective follow-up of outcome in major depression. Psychol Med. 1998 May; 28(3):731-5.

9 Thase ME, Buysse DJ, Frank E, Cherry CR, Cornes CL, Mallinger AG, et al. Which depressed patients will respond to interpersonal psychotherapy? The role of abnormal EEG sleep profiles. Am J Psychiatry. 1997 Apr; 154(4):502-9.

10 Perlis ML, Giles DE, Buysse DJ, Tu X, Kupfer DJ. Self-reported sleep disturbance as a prodromal symptom in recurrent depression. J Affect Disord. 1997 Feb;42(2-3):209_ 12.

11 Troxel WM, Kupfer DJ, Reynolds CF 3rd, Frank E, Thase ME, Miewald JM, et al. Insomnia and objectively measured sleep disturbances predict treatment outcome in depressed patients treated with psychotherapy or psychotherapy-pharmacotherapy combinations. J Clin Psychiatry. 2012 Apr;73(4): 478-85.

12 Gregory AM, Rijsdijk FV, Dahl RE, McGuffin P, Eley TC. Associations between sleep problems, anxiety, and depression in twins at 8 years of age. Pediatrics. 2006 Sep;118(3): 1124-32.

13 Modell S, Ising M, Holsboer F, Lauer CJ. The Munich vulnerability study on affective disorders: premorbid polysomnographic profile of affected high-risk probands. Biol Psychiatry. 2005 Nov;58(9):694-9.

14 Mongrain V, Lavoie S, Selmaoui B, Paquet J, Dumont $M$. Phase relationships between sleep-wake cycle and underlying circadian rhythms in Morningness-Eveningness J Biol Rhythms. 2004 Jun;19(3):248-57.

15 Serretti A, Benedetti F, Mandelli L, Lorenzi C, Pirovano A, Colombo C, et al. Genetic dissection of psychopathological symptoms: insomnia in mood disorders and CLOCK gene polymorphism. Am J Med Genet B Neuropsychiatr Genet. 2003 Aug;121B(1):35-8.

16 Utge SJ, Soronen P, Loukola A, Kronholm E, Ollila HM, Pirkola S, et al. Systematic analysis of circadian genes in a population-based sample reveals association of TIMELESS with depression and sleep disturbance. PLoS One. 2010 Feb;5(2):e9259.

17 Dmitrzak-Weglarz MP, Pawlak JM, Maciukiewicz M, Moczko J, Wilkosc M, Leszczynska-Rodziewicz A, et al. Clock gene variants differentiate mood disorders. Mol Biol Rep. 2015 Jan;42(1):277-88.

18 Ripke S, Wray NR, Lewis CM, Hamilton SP, Weissman MM, Breen G, et al.; Major Depressive Disorder Working Group of the Psychiatric GWAS Consortium. A mega-analysis of genome-wide association studies for major depressive disorder. Mol Psychiatry. 2013 Apr;18(4):497-511.
19 Hyde CL, Nagle MW, Tian C, Chen X, Paciga SA, Wendland JR, et al. Identification of 15 genetic loci associated with risk of major depression in individuals of European descent. Nat Genet. 2016 Sep;48(9):1031-6.

20 Bradfield JP, Taal HR, Timpson NJ, Scherag A, Lecoeur C, Warrington NM, et al.; Early Growth Genetics Consortium. A genomewide association meta-analysis identifies new childhood obesity loci. Nat Genet. 2012 May; 44(5):526-31.

21 Herbert D, Neves-Pereira M, Baidya R, Cheema S, Groleau S, Shahmirian A, et al. Genetic testing as a supporting tool in prescribing psychiatric medication: design and protocol of the IMPACT study. J Psychiatr Res. 2018 Jan; $96: 265-72$.

22 Beck AT, Steer RA, Brown GK. BDI-II: Beck depression inventory. Pearson; 1996.

23 Lingjaerde O, Ahlfors UG, Bech P, Dencker SJ, Elgen K. The UKU side effect rating scale. A new comprehensive rating scale for pschotropic drugs and a cross-sectional study of side effects in neuroleptic-treated patients. Acta Psychiatr Scand Suppl. 1987;334:1-100.

24 Arnarson TO, Olason DT, Smári J, Sigurethsson JF. The Beck Depression Inventory Second Edition (BDI-II): Psychometric properties in Icelandic student and patient populations. Nord J Psychiatry. 2008;62(5): 360-5.

25 Ha NT, Freytag S, Bickeboeller H. Coverage and efficiency in current SNP chips. Eur J Hum Genet. 2014 Sep;22(9):1124-30.

26 Purcell S, Neale B, Todd-Brown K, Thomas L, Ferreira MA, Bender D, et al. PLINK: a tool set for whole-genome association and population-based linkage analyses. Am J Hum Genet. 2007 Sep;81(3):559-75.

27 Anderson CA, Pettersson FH, Clarke GM, Cardon LR, Morris AP, Zondervan KT. Data quality control in genetic case-control association studies. Nat Protoc. 2010 Sep;5(9): 1564-73.

28 Clarke GM, Anderson CA, Pettersson FH, Cardon LR, Morris AP, Zondervan KT. Basic statistical analysis in genetic case-control studies. Nat Protoc. 2011 Feb;6(2):121-33.

29 Zondervan KT, Cardon LR. Designing candidate gene and genome-wide case-control association studies. Nat Protoc. 2007;2(10): 2492-501.

30 Auton A, Brooks LD, Durbin RM, Garrison EP, Kang HM, Korbel JO, et al.; $1000 \mathrm{Ge}-$ nomes Project Consortium. A global reference for human genetic variation. Nature. 2015 Oct;526(7571):68-74.

31 Team RC. A language and environment for statistical computing. R Foundation for Statistical Computing. 2013. Available from: http://www.r-project.org/.

32 Price AL, Zaitlen NA, Reich D, Patterson N. New approaches to population stratification in genome-wide association studies. Nat Rev Genet. 2010 Jul;11(7):459-63.

33 Howie BN, Donnelly P, Marchini J. A flexible and accurate genotype imputation method for the next generation of genome-wide association studies. PLoS Genet. 2009 Jun; 5(6):e1000529.

34 Delaneau O, Marchini J, Zagury JF. A linear complexity phasing method for thousands of genomes. Nat Methods. 2011 Dec;9(2):17981.

35 Verma SS, de Andrade M, Tromp G, Kuivaniemi H, Pugh E, Namjou-Khales B, et al. Imputation and quality control steps for combining multiple genome-wide datasets. Front Genet. 2014 Dec;5:370.

36 Nyholt DR, Low SK, Anderson CA, Painter JN, Uno S, Morris AP, et al. Genome-wide association meta-analysis identifies new endometriosis risk loci. Nat Genet. 2012 Dec; 44(12):1355-9.

37 Carlsson P, Kjellén L. Heparin biosynthesis. Handb Exp Pharmacol. 2012;207(207):23-41.

38 Pallerla SR, Lawrence R, Lewejohann L, Pan Y, Fischer T, Schlomann U, et al. Altered heparan sulfate structure in mice with deleted NDST3 gene function. J Biol Chem. 2008 Jun; 283(24):16885-94.

39 Aikawa J, Esko JD. Molecular cloning and expression of a third member of the heparan sulfate/heparin GlcNAc N-deacetylase/ N-sulfotransferase family. J Biol Chem. 1999 Jan; 274(5):2690-5

40 Malavaki CJ, Theocharis AD, Lamari FN, Kanakis I, Tsegenidis T, Tzanakakis GN, et al. Heparan sulfate: biological significance, tools for biochemical analysis and structural characterization. Biomed Chromatogr. 2011 Jan; 25(1-2):11-20.

41 Lencz T, Guha S, Liu C, Rosenfeld J, Mukherjee S, DeRosse P, et al. Genome-wide association study implicates NDST3 in schizophrenia and bipolar disorder. Nat Commun. 2013; 4(1):2739.

42 Meissner G. The structural basis of ryanodine receptor ion channel function. J Gen Physiol. 2017 Dec;149(12):1065-89.

43 Coumel P. Catecholaminergic-induced severe ventricular arrhythmias with AdamsStokes syndrome in children: report of four cases. Br Heart J. 1978;40:28-37.

44 Priori SG, Napolitano C, Tiso N, Memmi M, Vignati G, Bloise R, et al. Mutations in the cardiac ryanodine receptor gene (hRyR2) underlie catecholaminergic polymorphic ventricular tachycardia. Circulation. 2001 Jan;103(2): 196-200.

45 Pfeffer M, Müller CM, Mordel J, Meissl H, Ansari N, Deller T, et al. The mammalian molecular clockwork controls rhythmic expression of its own input pathway components. J Neurosci. 2009 May;29(19):611423.

46 Reppert SM, Weaver DR. Coordination of circadian timing in mammals. Nature. 2002 Aug;418(6901):935-41.

47 Debruyne JP, Noton E, Lambert CM, Maywood ES, Weaver DR, Reppert SM. A clock shock: mouse CLOCK is not required for circadian oscillator function. Neuron. $2006 \mathrm{May}$; 50(3):465-77. 
48 Evans DS, Parimi N, Nievergelt CM, Blackwell T, Redline S, Ancoli-Israel S, et al.; Study of Osteoporotic Fractures (SOF); Osteoporotic Fractures in Men (MrOS) Study Group. Common genetic variants in ARNTL and NPAS2 and at chromosome 12p13 are associated with objectively measured sleep traits in the elderly. Sleep (Basel). 2013 Mar;36(3): 431-46.

49 Soria V, Martínez-Amorós E, Escaramís G, Valero J, Pérez-Egea R, García C, et al. Differential association of circadian genes with mood disorders: CRY1 and NPAS2 are associated with unipolar major depression and CLOCK and VIP with bipolar disorder. Neuropsychopharmacology. 2010 May;35(6): 1279-89.
50 Lavebratt C, Sjöholm LK, Partonen T, Schalling M, Forsell Y. PER2 variantion is associated with depression vulnerability. Am J Med Genet B Neuropsychiatr Genet. 2010 Mar; 153B(2):570-81.

51 Byrne EM, Heath AC, Madden PA, Pergadia ML, Hickie IB, Montgomery GW, et al. Testing the role of circadian genes in conferring risk for psychiatric disorders. Am J Med Genet B Neuropsychiatr Genet. 2014 Apr;165B(3):254-60.

52 Jagannath A, Peirson SN, Foster RG. Sleep and circadian rhythm disruption in neuropsychiatric illness [Internet]. Curr Opin Neurobiol. 2013 Oct;23(5):888-94.

53 King DP, Takahashi JS. Molecular genetics of circadian rhythms in mammals. Annu Rev Neurosci. 2000;23(1):713-42.

54 Asher G, Gatfield D, Stratmann M, Reinke H, Dibner C, Kreppel F, et al. SIRT1 regulates circadian clock gene expression through PER2 deacetylation. Cell. 2008 Jul;134(2):317-28.
55 Lin L, Faraco J, Li R, Kadotani H, Rogers W, Lin $\mathrm{X}$, et al. The sleep disorder canine narcolepsy is caused by a mutation in the hypocretin (orexin) receptor 2 gene. Cell. 1999 Aug; 98(3):365-76.

56 Estabrooke IV, McCarthy MT, Ko E, Chou TC, Chemelli RM, Yanagisawa M, et al. Fos expression in orexin neurons varies with behavioral state. J Neurosci. 2001 Mar;21(5): 1656-62.

57 Lane JM, Liang J, Vlasac I, Anderson SG, Bechtold DA, Bowden J, et al. Genome-wide association analyses of sleep disturbance traits identify new loci and highlight shared genetics with neuropsychiatric and metabolic traits. Nat Genet. 2017 Feb;49(2):27481. 